Meta

Journal des traducteurs

Translators' Journal

\title{
Quo vadis, CIUTI?
}

\section{Martin Forstner}

Volume 57, numéro 1, mars 2012

La CIUTI, chef de file pour la promotion de l'employabilité et de la recherche

CIUTI: Leader in Advocating Employability and Research

URI : https://id.erudit.org/iderudit/1012737ar

DOI : https://doi.org/10.7202/1012737ar

Aller au sommaire du numéro

\section{Éditeur(s)}

Les Presses de l’Université de Montréal

ISSN

0026-0452 (imprimé)

1492-1421 (numérique)

Découvrir la revue

Citer cet article

Forstner, M. (2012). Quo vadis, CIUTI? Meta, 57(1), 8-22.

https://doi.org/10.7202/1012737ar

\section{Résumé de l'article}

Le présent article retrace le cheminement de la CIUTI depuis 50 ans, c'est-à-dire son développement en une organisation réputée ayant à son actif 40 membres en Europe et dans le monde. A ses débuts, la CIUTI avait certes comme but essentiel la promotion de la coopération entre ses membres (mobilité des étudiants et des enseignants, échange de connaissances), mais son autre objectif était de promouvoir la traductologie dans les universités en tant que discipline à part entière. L'auteur affirme qu'être membre de la CIUTI a une valeur symbolique qui confère, de ce fait, un capital social valorisant, sur les plans national et international, notamment en ce qui concerne le classement des instituts. En 1994, la CIUTI est devenue une association internationale régie par la loi belge ; en tant que telle, la CIUTI a voulu jouer son rôle en matière de formation afin d'influencer la politique langagière et traductionnelle européenne, en collaboration avec d'autres organisations internationales. Ses actions ont été facilitées par le fait que la CIUTI a aussi été déclarée ONG à Genève en 2003. Cependant, selon l'auteur, le développement interne de la CIUTI n'a pas suivi le même rythme que le rayonnement externe, indéniable, que cette association a connu et connaît encore en tant qu'important acteur international. Aussi, des modifications de la structure décisionnelle seront nécessaires, ainsi que la mise à disposition de moyens financiers liée à l'attribution de plus de pouvoir au Conseil de la CIUTI, de manière à permettre la mise en place d'une gestion professionnelle et efficace des affaires publiques. Une décision délicate incombe à la CIUTI : soit continuer à suivre le chemin déjà tracé et éviter toute forme de risque, soit prendre une voie nouvelle et accepter son rôle en tant qu'acteur clé dans notre ère de mondialisation.
Ce document est protégé par la loi sur le droit d'auteur. L’utilisation des services d’Érudit (y compris la reproduction) est assujettie à sa politique d'utilisation que vous pouvez consulter en ligne.

https://apropos.erudit.org/fr/usagers/politique-dutilisation/ 


\title{
LA CIUTI ET LE CONTEXTE INTERNATIONAL
}

\section{Quo vadis, CIUTI?}

\author{
MARTIN FORSTNER \\ Johannes Gutenberg-Universität Mainz, Germersheim, Germany \\ forstner@uni-mainz.de
}

\section{RÉSUMÉ}

Le présent article retrace le cheminement de la CIUTI depuis 50 ans, c'est-à-dire son développement en une organisation réputée ayant à son actif 40 membres en Europe et dans le monde. À ses débuts, la CIUTI avait certes comme but essentiel la promotion de la coopération entre ses membres (mobilité des étudiants et des enseignants, échange de connaissances), mais son autre objectif était de promouvoir la traductologie dans les universités en tant que discipline à part entière. L'auteur affirme qu'être membre de la CIUTI a une valeur symbolique qui confère, de ce fait, un capital social valorisant, sur les plans national et international, notamment en ce qui concerne le classement des instituts. En 1994, la CIUTI est devenue une association internationale régie par la loi belge; en tant que telle, la CIUTI a voulu jouer son rôle en matière de formation afin d'influencer la politique langagière et traductionnelle européenne, en collaboration avec d'autres organisations internationales. Ses actions ont été facilitées par le fait que la CIUTI a aussi été déclarée ONG à Genève en 2003. Cependant, selon l'auteur, le développement interne de la CIUTI n'a pas suivi le même rythme que le rayonnement externe, indéniable, que cette association a connu et connaît encore en tant qu'important acteur international. Aussi, des modifications de la structure décisionnelle seront nécessaires, ainsi que la mise à disposition de moyens financiers liée à l'attribution de plus de pouvoir au Conseil de la CIUTI, de manière à permettre la mise en place d'une gestion professionnelle et efficace des affaires publiques. Une décision délicate incombe à la CIUTI: soit continuer à suivre le chemin déjà tracé et éviter toute forme de risque, soit prendre une voie nouvelle et accepter son rôle en tant qu'acteur clé dans notre ère de mondialisation.

\begin{abstract}
This article shows that within 50 years CIUTI has developed into a reputable organization now with 40 member institutes both within and outside of Europe. In the beginning CIUTI was devoted to promoting the cooperation between its members (mobility of students and trainers; exchange of knowledge), but was also anxious to anchor translation studies at universities as a scientific discipline in its own right. The author shows that CIUTI membership is of symbolic value and provides important social capital thus being of advantage to its members in their national and international ranking. In 1994, CIUTI became an international association (association internationale) under Belgian law and then began to play a role in education matters to influence European language and translation politics in collaboration with other international organizations. This was made easier by the fact that CIUTI was registered as a NGO in Geneva in 2003. However, according to the author of this article, the internal development of CIUTI has not kept pace with the external requirements of becoming a global player. That will require changes in its decision making structure and in the disposal of its financial resources by giving the Board (Conseil) more power to implement an appropriately efficient and professional public affairs strategy. CIUTI has
\end{abstract}


a difficult decision to make, either to stay on the beaten track and avoid any risks or to forge a new future for itself and accept its role as a major global player.

\section{MOTS-CLÉS/KEYWORDS}

CIUTI, ONG, politique de la traduction, association internationale, mondialisation CIUTI, NGOs, translation politics, international association, globalization

\section{CIUTI - einst und jetzt}

Im Jahre 1960 trafen sich in Basel zum ersten Mal die Direktoren einiger „Sprachenund Dolmetscherschulen“, die den Universitäten Genf, Heidelberg, Paris-Sorbonne, Mainz (Germersheim), Saarbrücken und Triest angehörten, um anstehende Probleme bei der Ausbildung von Übersetzern und Dolmetschern zu diskutieren, Lösungen zu finden und die Zusammenarbeit zu erleichtern, denn letzteres war damals noch mit beträchtlichen Schwierigkeiten verbunden, vor allem was die Mobilität der Lehrenden und der Studierenden anging. Bereits beim zweiten Treffen (1961), bei dem auch ein Wiener Institut vertreten war, wurden Statuten vorgelegt, dann 1963 in Paris überarbeitet und schließlich 1964 in Triest verabschiedet für eine Vereinigung des Namens Conférence Internationale Permanente de Directeurs d'Instituts Universitaires pour la Formation de Traducteurs et d'Interprètes. Ihre Mitglieder wollten sich auf Hochschulebene für eine qualifizierende Ausbildung der Übersetzer und Konferenzdolmetscher einsetzen, die nach Abschluß ihres Studiums sofort einsetzbar sein sollten, wie die damaligen Guidelines for New Membership ausdrücklich betonten.

In den folgenden Jahren wurden in diesen Club der alljährlich tagenden Institutsdirektoren weitere Institute aufgenommen, doch legte man Wert darauf, den Kreis der Mitglieder klein zu halten. Dabei war und blieb das Interesse der CIUTI auf Westeuropa konzentriert, selbst als dann nordamerikanische Institute in Washington (1965), Montreal (1966) und Monterey (1988) hinzukamen. Diese Europalastigkeit hatte ihren Grund in der fortschreitenden wirtschaftlichen Integration in den Europäischen Gemeinschaften (seit 1991 Europäische Union) und dem zunehmenden Bedarf an Übersetzern und Dolmetschern für die Brüsseler Institutionen, was es schließlich notwendig und gerechtfertigt erscheinen ließ, der CIUTI einen angemessenen Rechtsstatus zu verschaffen, wie es dann am 24. November 1994 geschah, als sie unter der Bezeichnung Conférence Internationale Permanente d'Instituts Universitaires de Traducteurs et Interprètes als association internationale nach belgischem Recht eingetragen wurde.

Später dehnte sich das Netz der Mitgliedinstitute (siehe Verzeichnis in Annex 1), deren Zahl sich heute auf 40 beläuft, über Mittel- und Südeuropa aus, aber auch Institute außerhalb der Europäischen Union sind nunmehr vertreten, so in Beirut (1999), Seoul (2004), Minsk (2009), Sankt Petersburg (2006), Moskau (2010), Peking (2008), Shanghai (2009) und Guangdong (2011), denn die europäische Ausrichtung wurde inzwischen durch eine globale ergänzt, da sich die CIUTI der Nairobi Recommendation der Vereinten Nationen von 1976 verpflichtet fühlt, durch Übersetzungen zum Verständnis zwischen den Völkern beizutragen und deren Sprachenvielfalt zu erhalten, aber auch der Charta der Fédération Internationale des Traducteurs, die darauf abstellt, daß Übersetzen und Dolmetschen als eigenständige akademische Berufe weltweit anerkannt werden. Die CIUTI ist heute, nachdem sie 2003 in Genf beim Büro der Vereinten Nationen den Status einer NGO (non-governmental organization) erworben 
hat, bestrebt, mehr außereuropäische Mitglieder zu gewinnen, um ihre Ziele nachhaltig auf globaler Ebene verfolgen zu können.

\section{Im Interessengeflecht von Zusammenarbeit und Konkurrenz der CIUTI}

Die Mitgliedinstitute der CIUTI waren sich stets der Schwierigkeiten bewußt, die sich einer Zusammenarbeit entgegenstellten, da die Strukturen, die Inhalte, die Prüfungsanforderungen und die Dauer ihrer Studiengänge recht unterschiedlich waren. Angesichts dieser Divergenzen einigte man sich 1995 auf die Formel Gleichwertigkeit in Vielfalt (vgl. Forstner 1996: XIX), doch sollte eine Mitgliedschaft in der CIUTI die Qualität der Lehrpläne und der Lehrinhalte anzeigen sowie auf die Einhaltung rigoroser Prüfungsanforderungen hinweisen, wie es auch heute auf der CIUTI Website wie folgt ausgedrückt wird:

Equivalence in Diversity. CIUTI and its members aim to ensure a high quality in the training of translators and interpreters. In order to uphold this quality against the background of different national frameworks, CIUTI subscribes to the principle of equivalence in diversity. CIUTI does not seek uniformity in the degrees of translators and interpreters offered at its member institutions. Rather, the diverse structures of higher education in the countries of the CIUTI members should be exploited to ensure that the same quality standards are reached at the end of the degrees. Membership of CIUTI should signify to the outside world that the contents and the examination standards, as well as the knowledge, attitudes and skills are of equivalent quality.

Der akademische Status wurde stets durch die Notwendigkeit einer sowohl anwendungs- als auch forschungsgeleiteten Lehre gerechtfertigt, doch standen in den Anfangsjahren der CIUTI Probleme ganz anderer Art im Vordergrund, denn die Institute waren in den Universitäten, denen sie zugeordnet waren, zumeist deutlich getrennt von den Fremdsprachenphilologien wie Anglistik, Romanistik und Slawistik, die wenig geneigt waren, die Übersetzerausbildung als gleichwertig anzuerkennen, weshalb noch zwischen 1960 und 1975 viele Mitgliedinstitute damit befaßt waren, in ihren Hochschulen als eigene wissenschaftliche Disziplin mit der Bezeichnung Translationswissenschaft (Translation Studies) anerkannt zu werden, was dann in den 1980er und 1990er Jahren weitgehend gelungen war.

In diesem Kontext war es günstig, daß in den Organen der Europäischen Gemeinschaften (später Europäische Union) in großem Umfang übersetzt und in den Sitzungen der zahlreichen Kommissionen in alle offiziellen Sprachen gedolmetscht werden mußte. Dies trug dazu bei, daß an den Universitäten, denen die CIUTI-Institute angehörten, die Studiengänge Übersetzen und Dolmetschen zu Diplomstudiengängen aufgewertet wurden, um den Absolventen die rechtlichen Voraussetzungen für den Eintritt in den Brüsseler Beamten- und Angestelltenapparat zu schaffen.

Die fortschreitende wirtschaftliche Integration war mit bildungspolitischen Programmen (ERASMUS, TEMPUS, SOCRATES, usw.) verbunden, die das Zusammenwachsen Europas durch die Mobilität der Studierenden und der Lehrenden beschleunigen sollten, dies allerdings mit der Maßgabe, daß die Universitäten diesbezüglich zusammenarbeiten müßten, ansonsten sie von den bereitgestellten Finanzmitteln ausgeschlossen würden. Anders als erwartet schlossen sich die CIUTI-Mitgliedinstitute nicht zu einem übergreifenden europäischen „Netzwerk Übersetzen und Dolmet- 
schen“ zusammen, vielmehr bildeten sie Netzwerke mit allen möglichen anderen Universitäten, selbst wenn diese gar keine Übersetzer und Dolmetscher ausbildeten, sondern lediglich Fremdsprachenunterricht anboten. Auf diese Weise wurde jedes CIUTI-Institut zum Rivalen der anderen Institute, da die Verlockung, von den Finanzmitteln der EG zu profitieren, einfach zu groß war. Gerade zu dem Zeitpunkt, da sich die CIUTI als association internationale in Brüssel eingerichtet hatte, drohte bereits eine „Ent-Netzung“ derselben, die ihre Ursache zum einen im überzogenen Profilierungsstreben der Institute hatte, zum anderen aber auch darin, daß es gegensätzliche dogmatische Positionen hinsichtlich der Inhalte der Ausbildung gab, was die interne Kohäsion gefährdete, und schließlich auch deshalb, weil die Auffassungen differierten, wieweit eine CIUTI-Mitgliedschaft die Verpflichtung zur Zusammenarbeit und Solidarität auf der Basis eines do ut des verlange. Zwar war die CIUTI dank ihrem rechtlichen Status eine stabile Organisation, doch da sie aus selbständigen Instituten bestand, die sich in nur geringem Maße an Verhandlungsergebnisse, Abkommen oder Zusagen, die die Generalversammlung auf Empfehlung des Vorstands (conseil) erreicht hatte, binden lassen wollten, barg die CIUTI ein hohes Potential an innerer Polarisierung in sich, was die Herausbildung eine corporate identity erschwerte und ihrem Anspruch auf alleinige Interessenrepräsentation nach außen nicht gerade förderlich war. Man war sich aber darin einig, daß das Alleinstellungsmerkmal „CIUTI-Mitglied“ möglichst nicht gefährdet werden sollte, denn dies brachte bei den Experten der Eurokratie einen Pluspunkt ein bei Bewerbungen um Fördermittel.

Als die Anträge auf Mitgliedschaft gleichwohl zunahmen, wurde bei ihrer Behandlung das Kriterium Qualität in Lehre und Forschung in den Mittelpunkt gerückt bei den internen Diskussionen der CIUTI, was es dann notwendig machte, unter Verweis auf die Statuten der CIUTI ein geregeltes Aufnahmeverfahren zu implementieren. Dieser Vorgang der Verrechtlichung desselben brachte unvermeidlich eine gewisse Bürokratisierung mit sich, aber auch eine Versachlichung bei gleichzeitiger Verkürzung des Procedere (vgl. Antragsformular auf der CIUTI Website). Dennoch muß in der Regel selbst bei sehr zügiger Behandlung noch immer mit zwei Jahren gerechnet werden, was nicht zuletzt auch mit der Struktur der CIUTI zu tun hat, denn über die Aufnahme entscheidet letztendlich immer die Generalversammlung (dazu siehe unten 6). Trotz allem übt die CIUTI eine starke Anziehungskraft aus, was einer Erläuterung bedarf.

\section{Symbolischer Wert und soziales Kapital einer CIUTI Mitgliedschaft}

Die Hochschulforschung konstatiert das Phänomen des academic drift, worunter zu verstehen ist, daß Universitäten ihre Position im nationalen Ranking zu steigern suchen durch Einführung neuer Studiengänge oder durch Bildung von Netzwerken für spezielle Forschungsvorhaben mit anderen Universitäten, die bereits ein hohes Ranking haben, aber auch dadurch, daß ein Hochschulinstitut sich um Aufnahme in eine renommierte internationale Vereinigung bemüht, um an deren Reputation teilzuhaben und dadurch Wettbewerbsvorteile im Wettstreit der Hochschulen zu erzielen.

Letzteres war und ist für die CIUTI wichtig, denn ihr Akronym und ihr Logo sind zu internationalen Marken (brands) geworden, und als Vereinigung genießt sie bei den europäischen und zunehmend auch den außereuropäischen Hochschulen 
hohes Ansehen. Allerdings bietet die CIUTI als Symbol für anspruchsvolle akademische Ausbildung und erfolgreiche Forschung keine unmittelbaren wirtschaftlichen Vorteile, denn die Mitgliedschaft stellt ein nichtmaterielles Gut dar, dessen Wert umso höher ist, je beschränkter und je schwieriger der Zugang zu dieser Vereinigung ist. Aber der Aufwand und die Anstrengung, Mitglied in ihr zu werden, wird als lohnende Investition gesehen, denn im Erfolgsfall ergibt sich, wie es der französische Soziologe Pierre Bourdieu (1991: 73-75) bezeichnen würde, ein Gewinn an sozialem Kapital in Form von Reputation, Prestige und Distinktion, was die Zugehörigkeit zu einer nicht allen zugänglichen Gruppe oder Vereinigung, eben zur CIUTI, verschafft.

Die Reputation der CIUTI liegt in ihrem Ansehen innerhalb der Wissenschaftsgemeinschaft (scientific community), das auf den translationswissenschaftlichen Publikationen der Mitgliedinstitute, deren Lehrkörper über die notwendigen akademischen Qualifikationen verfügt, beruht, weshalb größter Wert auf den Nachweis der Forschungsleistungen eines jeden antragstellenden Instituts, das Mitglied werden will, gelegt wird. Im Zusammenhang mit der wissenschaftlichen Reputation wird in der CIUTI zudem darauf geachtet, daß die Personen, die sie nach außen vertreten, auch in der Translationswissenschaft (translation studies) anerkannte Gelehrte sind.

Das Prestige der CIUTI im Bereich der Ausbildung von Translatoren geht darauf zurück, daß die Mitgliedinstitute dank ihrer über Jahre hinweg erkennbar nachhaltigen Qualität der Lehre und der ihr zugrundeliegenden Forschungsleistungen hervorragen, was wiederum die Distinktion der CIUTI als Vereinigung gestärkt hat, sie von anderen Vereinigungen und Organisationen abhebt und unterscheidet und zum gesuchten Ansprechpartner macht bei professionellen Verbänden der Translatoren, bei Großunternehmen des Translationsmarktes und bei Bildungspolitikern, deren Sprachen- und Translationspolitik nicht nur in Europa, sondern weltweit den gesellschaftlichen Status der Übersetzer und Dolmetscher beeinflussen kann. Alle Mitgliedinstitute profitieren von diesen Kontakten der CIUTI zu den wichtigen Entscheidungsträgern in Wirtschaft und Politik, wozu auch seit 2004 die erfolgreichen CIUTI-Foren beitragen, die immer im Januar eines jeden Jahres in Genf in den Räumen der Vereinten Nationen veranstaltet werden.

Der symbolische Wert der CIUTI und ihr daraus resultierendes soziales Kapital darf, und insoweit herrscht Konsens unter den Mitgliedern, auf keinen Fall in Gefahr gebracht werden, weshalb es in ihrem Interesse sein muß, nur solche Mitglieder aufzunehmen, die ihrerseits bereits über eigenes soziales Kapital in ihrer nationalen Umgebung verfügen und die durch ihre Aufnahme in die CIUTI deren Wert vermehren. Das bringt es mit sich, daß bei neuen Mitgliedern nicht nur die Qualität von Forschung und Lehre nachzuweisen ist, sondern auch ihr vorhandenes nationales und internationales Ansehen. Darüber hinaus sollen sie auch bereit sein, sich damit in die CIUTI einzubringen, was eine permanente Beziehungsarbeit der Mitglieder untereinander erforderlich macht. Es kommt also darauf an, daß die neuen Mitglieder willens sind, durch ihre Mitarbeit Reputation und Distinktion der CIUTI zu wahren und zu mehren.

Die CIUTI hat wie jedes Symbol eine rein ideelle Seite, wie es in ihrer Mission, ihrem Leitbild und in den Werten, für die einzutreten die Mitglieder sich verpflichtet haben, zum Ausdruck kommt. Dies wird dann ergänzt durch eine Realsymbolik, in der sich dies konkretisiert im Tun und Handeln sowie in gemeinsamen Aktionen und Kampagnen. Zur Realsymbolik gehört aber auch die Nennung der Institute auf 
der CIUTI Website und die Verwendung des Logos der CIUTI auf Briefköpfen und auf Konferenzprogrammen, nicht zu vergessen solche Initiationshandlungen wie die Überreichung der Urkunde mit der CIUTI-Mitgliedschaft oder die erste Rede des Vertreters eines neuen Mitgliedinstituts vor der Generalversammlung. Dies alles stiftet Identität mit und Loyalität gegenüber der CIUTI, und verleiht gleichzeitig auch einer Interdependenzverpflichtung gegenüber allen Mitgliedern Ausdruck, was in seiner Wirkung nicht gering geschätzt werden sollte.

\section{Im Fokus: Qualität in Lehre und Forschung}

Eine weitere und sehr wichtig gewordene Form der Realsymbolisierung ist die durch die Mitgliedschaft zum Ausdruck gebrachte Garantie der Qualität der Ausbildung. Dies stellt die Zulassungskommission (admission commission) der CIUTI, die sich mit den Anträgen auf Mitgliedschaft zu befassen hat, vor nicht geringe Probleme, wie ihr Vorsitzender Hugo Marquant des öfteren der Generalversammlung der CIUTI dargelegt hat. Das CIUTI-Konzept der Qualität der Ausbildung vereine, so Hugo Marquant, zwei Elemente: die Qualität der Lehre (la qualité de la formation et de l'enseignement) und die ihr zugrunde liegende Forschung (la qualité de la recherche sous-jacente), die beide durch die Zulassungskommission überprüfbar seien, da sie sich konkretisierten einerseits als intrinsische Qualität (la qualité intrinsèque), wie sie das Institut jeweils intern als Lehrinhalte definiere und dann in der Ausbildung verwirkliche, andererseits als extrinsische Qualität (la qualité extrinsèque), wie diese Bemühungen von außen gesehen und beurteilt werden, unter anderem nach den Maßstäben der CIUTI. Man sei sich aber bewußt, daß die Qualität der Lehre zuvörderst von der Qualität der Forschung des jeweiligen Lehrkörpers abhänge, dessen Forschungsgegenstände mit dem Ausbildungsziel zusammenhängen und deren Ergebnisse in den Curricula verwertet werden müßten, weshalb denn auch Hannelore Lee-Jahnke, Präsidentin der CIUTI, von einem doppelten Praxisbezug spricht (LeeJahnke 2009: 136-138), was sich zum einen in der Einwirkung der Berufspraxis auf die translatorische Forschung, zum anderen auf die Lehre konkretisiere. Dies entspricht der Erkenntnis, daß eine Trennung von Grundlagenforschung und Angewandter Forschung heute obsolet geworden zu sein scheint (so Kaiser-Cooke 2003), und daß universitäre Forschung und ihre Wissensproduktion zunehmend im Kontext der Anwendung zu sehen sind, weshalb Hochschulen dazu beitragen, nicht nur neues Wissen zu produzieren, sondern dieses auch ohne große Zeitverluste an die Studierenden weiterzugeben und die Wege aufzuzeigen zur Nutzbarmachung und Verwertung dieses Wissens in der Gesellschaft (vgl. Campbell 2000: 139), was es erlaube, um es mit Hugo Marquant zu sagen, die Dichotomie von recherche fondamentale und recherche appliquée zu überwinden. Die Beurteilung des jeweiligen Verbundsystems von Lehre und Forschung bei einem antragstellenden Institut sei für die Zulassungskommission möglich, indem sie die vorgelegten Veröffentlichungen des Lehrkörpers einsehe, doch sei dann diese rein quantitative Betrachtung zu ergänzen durch eine Überprüfung durch Experten der CIUTI, wie sich vor Ort die Forschungsergebnisse qualitativ auf die Lehre auswirken.

Nicht in die Zuständigkeit der Zulassungskommission fallen jedoch, so Hugo Marquant, Fragen der Strategie der CIUTI als weltweit agierender Vereinigung mit nicht mehr eurozentrischer, sondern globaler Orientierung, der daran gelegen sei, 
unter Zugrundelegung ihres Leitbildes und ihrer Mission (vision \& mission), aber unter Berücksichtigung der lokalen und regionalen Gegebenheiten und Bedürfnisse in Asien, Afrika oder Lateinamerika, Kandidaten ausfindig zu machen, die neben ihrer qualitativen Eignung auch zur Reputation und Distinktion der CIUTI beitragen. Hier entsprechende Vorgaben zu machen, sei die Aufgabe des Vorstandes sowie der „Strategiegruppe der CIUTI“, letztendlich aber der Generalversammlung, deren Maxime es sein muß, das Überleben der CIUTI als international anerkannte und Einfluß ausübende Organisation zu gewährleisten. Daß dies wiederum mit besonderen Problemen verbunden ist, werden die nachfolgenden Ausführungen zeigen.

\section{Von einer bloßen Interessenvertretung zu einer advokatorischen Nichtregierungsorganisation (NGO)}

Die CIUTI wurde 1994 konzipiert als gemeinnützige Vereinigung (association sans but lucratif) nach belgischem Recht, in der nur Hochschulinstitute als juristische Personen Mitglied werden können. Sie verstand sich als Interessengruppe, die sich um die Belange ihrer Mitgliedinstitute kümmern sollte, zuvörderst um die Förderung der Qualität von Lehre und Forschung, um die Mobilität von Studierenden und Lehrenden, aber auch um die Zusammenarbeit mit anderen Organisationen, die ähnliche Ziel verfolgten, wie Artikel 3 der Statuten in der Fassung von 1994 zu entnehmen ist.

La CIUTI, qui est dénuée de tout esprit de lucre, a pour mission:

- d'harmoniser les programmes de cours et les examens en vue de faciliter la mobilité des étudiants entre les instituts membres et de permettre ainsi l'exploitation totale des ressources offertes du point de vue de la formation;

- de promouvoir et de développer les échanges d'étudiants et de professeurs;

- d'assurer la collaboration de ses membres dans le domaine de la recherche, dans le développement de nouvelles méthodes d'enseignement et d'instituer une assistance réciproque dans l'élaboration de documentation;

- d'instituer et de poursuivre une action concertée parmi ses membres pour ce qui concerne leurs relations avec les organisations internationales et leurs services linguistiques, les autres institutions à caractère international portant intérêt à la formation d'interprètes et de traducteurs, ainsi que les associations internationales d'interprètes et de traducteurs.

(CIUTI Statuten 1994: Artikel 3)

Diese damaligen Interessen ähneln denen einer Berufsorganisation, die die unterschiedlichen Interessen ihrer Mitglieder aggregiert, dann in deren Namen kollektiv handelt und sie in der Öffentlichkeit vertritt, wobei diese Interessenvertretung auch die Leitbilder und die Mission der Interessengruppe gegenüber der Gesellschaft und der politischen und wirtschaftlichen Öffentlichkeit zur Geltung bringt, wie es im letzten Absatz des Art. 3 (alte Fassung) beschrieben wird.

Bei der Statutenänderung von 2008 wurde dieser Artikel gründlich überarbeitet und lautet in der heutigen Fassung wie folgt:

La CIUTI, qui est dénuée de tout esprit de lucre, a pour mission:

- d'optimiser la qualité de la formation; de faciliter la mobilité des étudiants et des professeurs entre les instituts membres. 
- d'assurer la collaboration de ses membres dans le domaine de la recherche, dans le développement de nouvelles méthodes d'enseignement.

- de promouvoir les relations avec les organisations, les associations et les entreprises nationales et internationales, portant intérêt à la formation de professionnels de l'interprétation, de la traduction, de la communication multilingue et interculturelle.

(CIUTI Statuten 2008: Artikel 3)

Zwar sind als Hauptzwecke der CIUTI weiterhin die Qualität der Ausbildung, die Mobilität der Studierenden und der Lehrenden sowie die Zusammenarbeit in Forschung und Lehre aufgeführt, aber in Absatz 3 (neue Fassung) ist, was die Zusammenarbeit mit nationalen und internationalen Organisationen, Vereinigungen und Unternehmen anlangt, eine entscheidende Neuerung zu konstatieren der Gestalt, daß die Worte d'instituer et de poursuivre une action concertée parmi ses membres gestrichen worden sind, was so ausgelegt werden kann, daß die CIUTI als solche nunmehr zuständig sei für die Beziehungen zu den genannten Organisationen und daß sie bzw. der Vorstand (conseil) in diesem Bereich aktiv werden könne.

Dies ist im Zusammenhang zu sehen mit dem Umstand, daß es der CIUTI 2003 gelungen war, den Status einer non-governmental organisation (NGO) in Genf zu erhalten, weshalb sie als Teil der pluralistischen Interessenlandschaft international als Vermittlungsakteur auftreten kann, wofür sie dank ihrer auf Dauer angelegten Organisationsstruktur bestens geeignet ist, um Aufgaben, Themen und Probleme im Zusammenhang mit der Ausbildung von Übersetzern und Dolmetschern und der Stärkung ihrer gesellschaftlichen Position in allen Staaten zu artikulieren und auf diese Weise ein autonomes Diskursfeld zu konstituieren und dann auch eine diesbezügliche Diskurshoheit zu bewahren.

Diese Artikulationsfunktion (voice function) ist dann zu ergänzen durch die Funktion der Themensetzung (agenda setting) mit gleichzeitigem Beratungsangebot, weshalb die CIUTI unter diesem Aspekt als service NGO (Martens 2005: 31) anzusehen wäre, die mit anderen NGOs, IGOs und sonstigen Institutionen, die ebenfalls am Thema Translation interessiert sind, zusammenarbeitet. Letztendlich ist zu nennen die Funktion der Themenanwaltschaft (advocacy), was bedeutet, daß die CIUTI ganz allgemein die Interessen von Übersetzern und Dolmetschern wahrnimmt, indem sie Themen wie etwa die soziale Stellung der Translatoren in der Gesellschaft aufnimmt und ihnen internationale Aufmerksamkeit zu verschaffen versucht, weshalb sie dann zu den advocacy NGOs (dazu Martens 2005: 30; vgl. Gordenker und Weiss 1998: 32) zu zählen wäre insoweit, als sie als Vertreterin der ihr angehörenden Hochschulinstitute Kontakte zu den international governmental organizations (IGOs) wie den Vereinten Nationen und der Europäischen Union aufnimmt und pflegt, um auf diese Weise die politische Meinungsfindung und Meinungsbildung nicht nur im Interesse ihrer Mitglieder, sondern aller ausbildenden Institute zu beeinflussen.

Sich als service and advocacy NGO zu betrachten, eine eigene Organisationsidentität zu entwickeln, dann einem gemeinsamen code of conduct zu folgen und dementsprechend nachhaltig zu agieren, erfordert ein Umdenken der CIUTIMitgliedinstitute, denn es geht nicht mehr allein darum, die Interessen der eigenen Organisation im Auge zu haben sowie deren Größe und Distinktion zu steigern, vielmehr ist damit verbunden eine Vergrößerung der eigenen Aktionsreichweite, die sich nicht beschränkt auf die Zusammenarbeit der einzelnen Mitgliedinstitute bei 
translationswissenschaftlichen Einzelprojekten, so wichtig dies auch sein mag für den internen Zusammenhalt der CIUTI, sondern die den Anspruch erhebt, zuständig zu sein für die Ausbildung von Übersetzern und Dolmetschern weltweit.

Möglicherweise überfordert dies die Vorstellungskraft einiger CIUTI-Mitgliedinstitute, für die es schon vor 20 Jahren mental schwer zu verkraften war, daß sich die CIUTI auf die Brüsseler Bühne begab, die sich dann aber damit abfanden und die es sich nun bequem eingerichtet haben im europäischen Hochschulraum unter dem Patronat der Europäischen Kommission mit ihren finanziellen Anreizen, weswegen ihre Bereitschaft, sich mit den Herausforderungen der außereuropäischen Translationswelt auseinanderzusetzen, begrenzt bleibt. Doch wenn die CIUTI diesen Schritt wagen sollte, dann würde sie tatsächlich zu einem global player. Wie alle NGOs (dazu Frantz und Martens 2006: 15) muß sie dann aber ihre Kampagnenfähigkeit unter Beweis stellen, um als Partner attraktiv zu bleiben, um ihre Vorstellungen auf internationaler Ebene zu platzieren und damit öffentliche Aufmerksamkeit zu gewinnen, sei es in Zusammenarbeit mit den jeweiligen nationalen und internationalen Berufsverbänden und der Translationsindustrie, sei es mit den Vereinten Nationen und der Europäischen Kommission, bei denen sie dann in der Rolle eines agenda and standard setter die Probleme der Ausbildung von Übersetzern und Dolmetschern immer wieder vorbringt, sie auf die Tagesordnung der Vereinten Nationen bzw der supranationalen Europäischen Union zu setzen versucht und als Schlüsselakteur (key actor) daran mitarbeitet, internationale Standards zu setzen oder deren Formulierung wenigstens zu beeinflussen. Die Vertreter der CIUTI müßten dann fungieren als Zulieferer von Informationen und als policy-Berater, sich am Entwurf von successful best practice standards beteiligen, oder bei der Implementierung und Verbreitung derselben mitwirken. Auf diese Weise würden sie das erfüllen, was nach Frantz / Martens (2006: 49). NGOs sind, nämlich formale (professionalisierte) unabhängige gesellschaftliche Akteure, deren Ziel es sei, progressiven Wandel und soziale Anliegen auf der nationalen oder der internationalen Ebene zu fördern.

Nun ist aber einzuräumen, daß derartige Versuche der CIUTI in der Vergangenheit nur teilweise erfolgreiche Interaktionen zeitigten, wie im Falle der Implementierung des European Master in Conference Interpreting (vgl. dazu Forstner 2010: 199) und des European Master in Translation, bei denen zwar CIUTI-Institute mitwirkten, aber die CIUTI als Organisation nicht in Erscheinung trat. Auf Seiten der CIUTI sollte man in Zukunft darauf hinwirken, expressis verbis als Partner genannt zu werden, wenn es sich um Projekte, Koalitionen oder Netzwerke handelt, in die mehrere CIUTI-Mitgliedinstitute eingebunden sind. Wenn die CIUTI in Zukunft bei derartigen Fällen in Form einer zumindest semiformellen Kooperation beteiligt sein will, dann muß sie selbstbewußt und eindeutig mit dem Anspruch auftreten, zuständig zu sein für die Ausbildung von Übersetzern und Dolmetschern weltweit, was sie dann notwendigerweise in Konkurrenz zu anderen Organisationen treten läßt, die dies ebenfalls beanspruchen, so etwa zur Fédération des Traducteurs oder zum European Language Council, ganz zu schweigen von den zahlreichen nationalen Berufsverbänden, doch ließe sich dies durch Zusammenarbeit regeln. Diese Organisationen dürften einer Partnerschaft nicht abgeneigt sein, da auch ihr Erfolg zumeist auf ihre Fähigkeit zurückzuführen ist, mit anderen Organisationen transnationale Netzwerke zu bilden, wobei der Umfang einer Zusammenarbeit recht unterschiedlich ausfallen und von gelegentlichem Informationsaustausch bis hin zu enger Koopera- 
tion bei der Durchführung von gemeinsamen Projekten reichen kann (vgl. dazu Curbach 2003: 65).

Die Vorteile einer solchen Vernetzung werden darin liegen, daß

der Informationsaustausch über bestimmte Themen erleichtert und gefördert wird und die geografische Reichweite und der erzeugbare politische Druck sich vergrößern. Durch den Zusammenschluß zu Netzwerken steigt so vor allem für kleinere oder ressourcenarme NGOs die Möglichkeit der Einflussnahme (Curbach 2003: 66),

wie etwa bei der CIUTI, der daran gelegen sein müßte, die bisherigen kurzfristigen Koalitionen, etwa mit der Fédération des Traducteurs, durch stabilere Formen mit stärkeren Bindungen zu ersetzen. Eine solche dichte Vernetzung könnte die Effektivität der CIUTI erhöhen, denn „Networks operate best when they are dense, with many actors, strong connections among groups in the network, and reliable information flows" (Keck und Sikkink 1998: 28).

Das könnte sich auf die Beteiligung der CIUTI an Kooperations-, Verhandlungsund Aktionsnetzwerken positiv auswirken, so auch in der Europäischen Union, wo es die Europäische Kommission seit geraumer Zeit vorzieht, mit möglichst weit vernetzten Organisationen, die im Brüsseler Jargon als Euro-groups bezeichnet werden, zu interagieren, denn a well organized Euro-group can make itself indispensable to the Commission by bringing representative opinion and other resources from one source (Greenwood 1997: 4; auch Freres 2000: 135), wie es der Fall ist beim European Language Council, dessen alerter Vorstand auf die europäische Sprachenpolitik beträchtlichen Einfluß ausübt und unter Rückgriff auf die Expertise seiner Mitglieder, die die Lage in allen europäischen Ländern kennen, als Berater nachgefragt ist, weshalb er seine eigenen Vorhaben effektiv und effizient den maßgeblichen Entscheidungsträgern in den Generaldirektionen der Europäischen Kommission vermitteln kann. Eine noch engere Zusammenarbeit der CIUTI mit einer solchen Euro-group wie dem European Language Council, dem die CIUTU ja auch angehört, sollte deshalb hohe Priorität genießen.

Auch die Vereinten Nationen vergeben bekanntlich ihren offiziellen Beraterstatus vorzugsweise an größere Organisationen oder an solche mit großen Netzwerken (vgl. Curbach 2003: 67; Gordenker und Weiss 1998: 33; Martens 2005: 19), wie etwa an sog. NGO-Multis wie Greenpeace oder an große Koalitionen, die straff organisiert und strukturiert sind, weshalb das letztendliche Ziel für die CIUTI sein müßte, ein solcher NGO-Multi mit einer eigenen corporate identity für den Themenbereich Übersetzen, Dolmetschen und sonstige Sprachendienstleistungen $\mathrm{zu}$ werden, was allerdings umfangreiche Investitionen in die eigene Organisation notwendig machen würde.

\section{Innovationspotentiale benötigen effiziente Entscheidungsstrukturen}

Bei allen NGOs hängt der Erfolg von einer gelungenen Professionalisierung der internen Führungs- und Entscheidungsstrukturen ab, allerdings kommt es darauf an, um welche Art von NGO es sich handelt, denn zum einen gibt es NGOs, die von ihren Mitgliedern nur unterstützt werden (supported by their members), zum anderen solche, die ihren Mitgliedern gehören (possessed by their members) (dazu Anheier und Themudo 2002: 307). Bei den Organisationen, die von ihren Mitgliedern lediglich unterstützt werden, haben diese keinen oder doch nur geringen Einfluß auf die 
Führungsprozesse im Vorstand, denn sie stellen in erster Linie die vorzugsweise finanziellen Ressourcen zur Verfügung. Dank einer solchen Struktur hat der Vorstand die Macht, eigenständig Entscheidungen zu treffen, was eine zweckdienliche, zielgerichtete und vor allem effiziente Führung ermöglicht. Bei der zweiten Art, die als den Mitgliedern gehörend zu bezeichnen wäre, stellt die gesamte Struktur auf die Mitglieder und ihr umfassendes Stimmrecht ab, wobei der Führung des Vorstands und seinen Entscheidungen Mißtrauen entgegen gebracht wird, weshalb diese in der Regel jeweils durch die Generalversammlung zu genehmigen sind.

Die CIUTI ist sicherlich der letzteren Gruppe zuzurechnen, wobei die Mitgliedschaft im Mittelpunkt steht, da sich in ihr der enorme Symbolwert verkörpert und sie die Quelle des angestrebten sozialen Kapitals ist (wie oben in 3 dargelegt). Dieser Umstand bringt es mit sich, daß in den Generalversammlungen der CIUTI seit Jahrzehnten viel Zeit darauf verwendet wird, über die Anträge auf Mitgliedschaft zu diskutieren, da man auf keinen Fall dem Vorstand (conseil) die alleinige Entscheidung überlassen möchte.

Diese Konstruktion kann sich auf die Arbeit des Vorstands hinderlich und negativ insofern auswirken, als der Raum für selbständige Aktionen und Initiativen im Bereich des agenda setting, also der Beeinflussung von Politik-Formulierung und Politik-Implementierung, sehr eingeschränkt wird, da kaum weitreichende Verpflichtungen eingegangen werden können, vielmehr alles unter dem Vorbehalt der Genehmigung durch die nächste Generalversammlung steht, die jeweils im Mai eines Jahres zusammentritt, weshalb wegen solcher eingeschränkten Handlungsspielräume des Vorstands die vorhandenen Innovationspotentiale nicht voll und auch nicht effizient genutzt werden.

Wegen dieser umständlichen Beschlußfassungsstrukturen kann die CIUTI als Akteur ihre thematischen Ziele und Interessen unter Ausnutzung aller sich bietenden Chancen nicht verfolgen und ist deshalb auch kaum in der Lage, Einfluß zu nehmen im internationalen Gefüge, sei es in der Europäischen Union, sei es bei den Vereinten Nationen oder sei es bei den internationalen Berufsorganisationen wie FIT und AIIC, oder sei es bei den weltweit operierenden Translationsunternehmen. Will man diese derzeitige mitgliederorientierte Ausrichtung beibehalten, aber gleichwohl effizient auf internationaler Ebene mitgestalten, so muß, womöglich durch Änderung der Statuten, der Vorstand mit größerer Gestaltungsmacht und weitgehenden Entscheidungsbefugnissen jenseits der täglichen operationellen Geschäfts ausgestattet werden, um eine nachhaltige Wirkung der CIUTI, etwa durch selbständig erarbeitete Grundsatzpapiere, die rechtzeitig veröffentlicht und als Pressemitteilungen versandt werden, sicherzustellen.

\section{Nachhaltiges Handeln trotz finanziellen Restriktionen}

In jeder Organisation kommt irgendwann der Zeitpunkt, daß die umfangreicher werdenden Aufgaben und die größer gewordene Zahl der Mitglieder den Übergang von ehrenamtlicher zur hauptamtlicher Mitarbeit im Vorstand erforderlich machen, um die Funktionsfähigkeit sicherzustellen (vgl. dazu die Ausführungen von Frantz / Martens 2006 mit einschlägiger Fachliteratur), denn auch non-profit organizations mit gemeinnütziger Orientierung müssen ökonomisch denken und ihre finanziellen Möglichkeiten bei ihren strategischen Überlegungen einbeziehen. 
Dies erfordert eine klare Festlegung der Ziele, die man erreichen will, was ein komplexer und oft schmerzhafter Prozess ist, bei dem unter Beachtung des KostenNutzen-Verhältnisses Prioritäten zu setzen sind, denn ohne ausreichende finanzielle Ressourcen sind die schönsten Vorhaben zum Scheitern verurteilt. Auch müssen rechtzeitig die Chancen der Akzeptanz einer neuen Strategie durch die Mitglieder berücksichtigt werden, denn die interne Kohäsion einer Organisation hängt weitgehend von deren Bereitschaft zur tatsächlichen Umsetzung der vorgeschlagenen Projekte ab.

Selbst wenn eine Profitmaximierung und das Ansammeln von Kapital nicht die Ziele der CIUTI sind, sondern die Durchsetzung ihres formulierten Anliegens, eine Interessenvertretung der Translatoren ausbildenden Hochschulinstitute zu sein, so müssen die finanziellen Möglichkeiten immer berücksichtigt werden. Dazu gehört, daß die regelmäßige Finanzierung der CIUTI durch Mitgliedsbeiträge gesichert sein muß, weshalb, unter Beachtung der oben genannten Kautelen hinsichtlich der Eignung der antragstellenden Institute, die Mitgliederzahl noch weiter steigen muß, um eine Verstetigung des finanziellen Zuflusses zu erreichen. Auf diese Weise würde die Gefahr verringert, daß dadurch, daß ein Mitgliedinstitut aus irgendeinem Grund, etwa einer plötzlichen Laune seines Direktors folgend, den Austritt erklärt, die Organisation als solche in eine finanzielle Schieflage gerät.

Dennoch wird die CIUTI weiterhin eine low budget organization bleiben, in der bei einem jährlichen Mitgliedsbeitrag von bescheidenen 250 Euro die Aufgaben des Vorstands (conseil) „ehrenamtlich“ erfüllt werden müssen, weshalb Präsident, Vizepräsidenten, Generalsekretär und Schatzmeister keine Angestellten der CIUTI sind, selbst wenn sie durch die Generalversammlung ad personam für drei Jahre gewählt werden. Ihnen werden bisher auch keine Reise- und Hotelkosten erstattet, denn wie sie ihre Teilnahme an den notwendigen Sitzungen finanzieren, ist ihre Angelegenheit. Als Professoren haben sie an ihren Hochschulen ihre Lehr- und Forschungsaufgaben zu erfüllen, was bedeutet, daß sie für die Angelegenheiten der CIUTI Zeit aufbringen, die sie eigentlich ihrer Forschung widmen sollten. Da sie in der Regel nicht als vermögend bezeichnet werden können, stehen sie bei Ausübung ihrer CIUTI-Ämter unter dem Einfluß ihrer Institute, was, trotz aller Beteuerungen des Gegenteils, die Gefahr mit sich bringt, daß sie die Interessen ihrer Institute - bewußt oder unbewußt - zu denen der CIUTI machen. Um dies etwas zu mindern und um die Aktionsmöglichkeiten des Vorstands zu erweitern, wäre die CIUTI gut beraten, dem Vorstand, aber auch den Mitgliedern der Strategiekommission für deren Schlüsselaktionen oder den sonstigen ad hoc gebildeten Expertengruppen die Reisekosten zu erstatten, nach dem Vorbild der Regelung für die Zulassungskommission, denn externen Gutachtern kann nicht zugemutet werden, neben ihrer unentgeltlichen Gutachtertätigkeit auch noch selbst die Reise- und Hotelkosten zu bezahlen.

Eine solche Verwendung der Mitgliedsbeiträge wäre zu vertreten, ansonsten man tatsächlich zum „Altherrensystem“ der Frühzeit der CIUTI zurückkehren würde, mit der Folge, daß für den Vorstand nur solche Kandidaten in Frage kommen, hinter denen vermögende Institute stehen. Ohne über selbst auferlegte Verhaltensregeln (codes of conduct) zu verfügen und ohne jemals Zuständigkeiten festgelegt zu haben, ist die CIUTI gegenwärtig auf ein verträgliches Miteinander ihrer ehrenamtlich tätigen Vorstandsmitglieder angewiesen, denn budgetäre Restriktionen lassen keine im Management ausgebildeten hauptamtlichen Kräfte für 
spezielle Geschäftsbereiche zu, aber die vorgeschlagene Minimalreform würde es dem Vorstand (conseil) ermöglichen, ein bescheidenes Public Affairs Management zu betreiben im Umgang mit anderen Vereinigungen wie dem European Language Council, vor allem mit den mächtigen Akteuren auf der Ebene des Europäischen Parlaments und der Europäischen Kommission mit ihrer politischen Bürokratie in den Generaldirektionen Übersetzen und Dolmetschen. Da die CIUTI anders als die Lobbyisten der Wirtschaft nicht auf große finanzielle Ressourcen zurückgreifen kann, wird nichts anderes übrig bleiben, als sich weiterhin auf die persönlichen Kontakte des Vorstands, die in mühevoller Arbeit in den letzten Jahren aufgebaut worden sind, zu verlassen.

\section{Ausblick: Zu neuen Ufern}

Innerhalb von 50 Jahren hat die CIUTI sich stark verändert. Gegenwärtig steht sie vor der Entscheidung, ob sie den herkömmlichen Weg einfach fortsetzen und dabei jedes Risiko vermeiden will, oder ob sie einen Weg einschlagen soll, der verlangt, daß sie ihre Rolle als globaler Akteur voll akzeptiert.

Die Mitgliedinstitute der CIUTI sollten aber angesichts der dargelegten finanziellen Restriktionen keine überhöhten Erwartungen hegen, vielmehr die realen Wirkungsmöglichkeiten des Vorstands und seine Grenzen erkennen, was auch mit einer Ent-Idealisierung der Vereinigung verbunden sein könnte. Andererseits bietet sich die Möglichkeit, die Irrelevanzfalle zu vermeiden und neue Bedeutung durch Umgestaltung zu gewinnen, indem man die bisherige Rolle einer rein berufsorientierten Interessengruppe erweitert um die Komponente einer global advokatorisch handelnden Nichtregierungsorganisation.

\section{LITERATUR}

Anheier, Helmut und Themudo, Nuno (2002): Führung und Management von Internationalen Mitgliederorganisationen. In: Christiane Frantz und Annette Zimmer, eds. Zivilgesellschaft international. Alte und neue NGOs. Opladen: Leske + Budrich, 304-325.

Bourdieu, Pierre (1991): Language and Symbolic Power. Oxford: Blackwell.

CAmpBell, David F. J. (2000): Forschungspolitische Trends in wissenschaftsbasierten Gesellschaften. Strategiemuster für entwickelte Wirtschaftssysteme. Wirtschaftspolitische Blätter. 47(2):130-143.

Curbach, Janina (2003): Global Governance und NGOs. Transnationale Zivilgesellschaft in internationalen Politiknetzwerken. Opladen: Leske + Budrich.

Forstner, Martin (1996): Zur Übersetzer- und Dolmetscherausbildung. In: Martin Forstner, ed. C.I.U.T.I Translation and Interpreting Studies 1995. Bamberg, XVII-XXIII.

Forstner, Martin (2010): Zur Rolle der Generaldirektion Dolmetschen der Europäischen Kommission als europäischer Marktführer. In: Nadja Grbić, Gernot Hebenstreit und Gisella Vorderobermeier, et al., eds. Translationskultur revisited. Festschrift für Erich Prunč. Tübingen: Stauffenburg, 190-203.

Frantz, Christiane und Martens, Kerstin (2006): Nichtregierungsorganisationen (NGOs). Wiesbaden: Verlag für Sozialwissenschaften.

Freres, Christian L. (2000): Striving for influence in a complex environment: NGO advocacy in the European Union. In: Paul Lewis und Tina Wallace, eds. New Roles and Relevance. Development NGOs and the Challenge of Change. Bloomfield: Kumarian Press, 131-138.

Gordenker, Leon und Weiss, Thomas G. (1998): Devolving responsibilities: a framework for analysing NGOs and Services. In: Thomas G. WeIss, ed. Beyond UN subcontracting. Task- 
sharing with regional security arrangements and service-providing NGOs. London: Macmillan, 30-35.

Greenwood, Justin (1997): Representing Interests in the European Union. London: Macmillan. KAISER-COOKE, Michèle (2003): Translation, Evolution und Cyberspace: Eine Synthese von Theorie, Praxis und Lehre. Frankfurt am Main, Wien: Peter Lang.

Keck, Margaret E. und Sikкink, Kathryn (1998): Activists beyond Borders. Ithaca: Cornell University Press.

LeE-JAhnke, Hannelore (2009): Doppelter Praxisbezug und Kompetenzvermittlung als Problem der Qualitätssicherung translatorischer Studiengänge. In: Martin Forstner, Hannelore Lee-Jahnke und Peter A. Schmitt, eds. CIUTI-Forum 2008. Enhancing Translation Quality: Ways, Means, Methods. Bern: Peter Lang, 133-193.

MARTENS, Kerstin (2005): NGOs and the United Nations. Institutionalization, professionalization and adaptation. Houndmills/Basingstoke: Palgrave Macmillan.

\section{ANNEX}

\section{CIUTI member institutes}

Faculté de Traduction et d'Interprétation, Université de Genève (CH) (1960)

Ecole Supérieure d'Interprètes et de Traducteurs (ESIT) - Université Paris III - Sorbonne Nouvelle (F) (1960)

Seminar für Übersetzen und Dolmetschen, Universität Heidelberg (D) (1960)

Fachbereich Translations-, Sprach- und Kulturwissenschaft (Germersheim), Universität Mainz

(D) (1960)

Angewandte Sprachwissenschaft sowie Übersetzen und Dolmetschen, Universität des Saarlandes (D) (1960)

Scuola Superiore di Lingue Moderne per Interpreti e Traduttori, Università degli Studi di Trieste (I) (1960)

Zentrum für Translationswissenschaft, Universität Wien (A) (1961)

Division of Interpretation and Translation, Georgetown University, Washington D.C. (1965, suspended in 2003)

Département de linguistique et de traduction, Université de Montréal (Canada) (1966)

Department of Translators and Interpreters, Artesis University College Antwerp (B) (1967)

Faculté de Traduction et d'Interprétation, École d'Interprètes Internationaux, Université de Mons-Hainaut (B) (1969)

Department of European Studies and Modern Languages, University of Bath (UK) (1971)

Faculty of Modern Languages, Copenhagen Business School (DK) (1971)

Department of Languages and Intercultural Studies, Heriot-Watt University (Edinburg) (UK) (1976)

Institut Supérieur d'Interprétation et de Traduction (ISIT) (Paris) (F) (1980)

Department of Language and Business Communication, Aarhus School of Business (DK) (1981)

Department of Modern Languages, University of Bradford (UK) (1986, suspended in 2008)

Graduate School of Translation, Interpretation and Language Education, Monterey Institute of International Studies (USA) (1988)

Institut Supérieur de Traducteurs et Interprètes (ISTI), Haute École de Bruxelles (B) (1991)

Institut für Translationswissenschaft, Universität Innsbruck (A) (1994)

Institut für Theoretische und Angewandte Translationswissenschaft, Universität Graz (A) (1995)

Departement Vertaalkunde- Faculty of Translation Studies, Hogeschool Gent - University College Ghent (B) 1996

Institut für Angewandte Linguistik und Translatologie (IALT), Universität Leipzig (D) 1998 Institute of Translation Studies, Charles University (Prague) (CZ) (1998)

Scuola Superiore di Lingue Moderne per Interpreti e Traduttori (Forli), Università degli Studi di Bologna (I) (1999) 
Ecole de Traducteurs et d'Interprètes de Beyrouth, Université Saint-Joseph (LB) (1999)

Departement Toegepaste Taalkunde, Lessius (Antwerp) / KU. Leuven (B) (2000)

Institut Libre Marie Haps, Haute École Léonard de Vince, Bruxelles (B) (2000)

Facultad de Ciencias Humanas y Sociales, Traducción e Interpretación, Universidad Pontificia Comillas (Madrid) (E) (2000)

School of Social Sciences, Humanities and Languages, University of Westminster (UK) (2002)

Graduate School of Interpretation and Translation, Hankuk University of Foreign Studies, Seoul (South Korea) (2004)

Department of Translation and Interpreting, University of Ljubljana (SL) (2004)

Institut für Translation und Mehrsprachige Kommunikation, Fachhochschule Köln (D) (2005)

Institut de Traduction et d'Interprétation, Sankt Peterburg State University (Russia) (2008)

Graduate School of Translation and Interpretation, Beijing Foreign Studies University (China) (2008)

Facoltà di Interpretariato e Traduzione, Libera Università San Pio V (Roma) (I) (2008)

Minsk State Linguistic University (Belarus) (2009)

Graduate Institute of Interpretation and Translation, Shanghai International Studies University (China) (2009)

School of Translation and Interpretation (MSU), Lomonosow Moscow State University (Russia) (2010)

Facultad de Traducción e Interpretación, Universidad de Granada (E) (2010)

Faculty of Humanities, Arts, Languages and Education, London Metropolitan University (UK) (2010)

School of Interpreting and Translation Studies, Guangdong University of Foreign Studies (China) (2011)

\section{Associated Members}

Norwegian School of Economics and Business Administration, Bergen (N) (2001)

Association Internationale des Interprètes de Conférence (AIIC), Genève (CH) (2007)

Bundesverband der Dolmetscher und Übersetzer, Berlin, (D) (2009)

Translators Association of China (TAC), Beijing (China) (2011) 